Suite à d'importants travaux, la nouvelle ordonnance sur la formation des assistantes médicales a pu être mise en vigueur avec succès. Cette ordonnance et le plan de formation forment la base du nouvel «Aide-mémoire pour les assistantes médicales» créé par le Dr Emil Schalch, président des délégués cantonaux aux questions des assistantes médicales. Il s'agit, en l'occurrence, d'un excellent outil pédagogique. Nous saisissons l'occasion pour le remercier vivement de cet important travail réalisé parallèlement à son activité de libre praticien.

Dr Ernst Gähler, vice-président de la FMH, Responsable du domaine Paramedics

\title{
Le cabinet médical au centre du nouvel aide-mé- moire pour la formation des assistantes médicales
}

Interview: Fabienne Hohl

*ertaines pages du nouve aide-mémoire pour les assistantes médicales peuvent être consultées en ligne sur le site internet de la FMH: www.fmh.ch $\rightarrow$ Services $\rightarrow$ Assistantes médicales
Souhaité depuis longtemps et conçu de manière à répondre aux exigences de la pratique, le nouvel aidemémoire pour les assistantes médicales* arrive à point nommé pour la rentrée scolaire et l'entrée en vigueur de la nouvelle ordonnance de formation des assistantes médicales. Il s'adresse tant aux formateurs qu'aux apprenants dans le sens où il propose des «thèmes hebdomadaires» pour les travaux avec le formateur et des chapitres pour l'étude individuelle. L'autre nouveauté est que le cabinet médical devient ainsi un lieu de formation équivalent aux écoles professionnelles et aux cours interentreprises. Le cabinet de praticien revêt ainsi l'importance qui lui revient et qui va au-delà de l'exercice d'activités isolées. Le travail d'équipe et les interfaces entre le travail des assistantes et du médecin sont revalorisés en conséquence. Les connaissances médicales de base sont transmises par le nouvel aide-mémoire dans la mesure où elles sont nécessaires à la compréhension des processus de travail au cabinet.

La structure de l'aide-mémoire s'inspire de l'activité quotidienne dans un cabinet médical: tâches administratives, contacts avec les assureurs, prise en charge du patient, laboratoire, situation de formation et d'apprentissage au cabinet médical. Il tient compte des objectifs de formation fixés dans le nouveau plan de formation des assistantes médicales élaboré par l'Office fédéral de la formation professionnelle et de la technologie (OFFT). Mais il ne faut pas confondre l'aide-mémoire destiné aux assistantes médicales avec la documentation de formation (anciennement Journal de travail). Le premier a en effet pour but d'axer les études sur la pratique et le second permet aux apprenties de relever leurs activités quotidiennes, expériences et progrès.

Le nouvel aide-mémoire pour les assistantes médicales a été rédigé par le Dr Emil Schalch, maître d'apprentissage chevronné et membre de la commission de réforme pour le plan de formation des assistantes médicales. Détenteur d'un cabinet médical à la campagne, le Dr Schalch, qui s'est penché sur la question des processus au cabinet et des tâches des assistantes médicales dans le cadre de la gestion de la qualité des soins, explique, dans l'entretien ci-après, les tenants et les aboutissants de ce nouvel outil pédagogique.

\section{«Réfléchir en équipe et assumer une responsabilité commune dans les tâches quotidiennes au cabinet»: interview avec Emil Schalch}

Quelle est la particularité principale de cet aide-mémoire? Emil Schalch: Il est extrêmement bien adapté à la pratique et s'éloigne de la conception selon laquelle le cabinet médical n'est qu'un lieu pour mettre en pratique et s'exercer, pendant que l'école professionnelle enseigne exclusivement la théorie et que les cours interentreprises transmettent les compétences pratiques. La formation en cabinet peut et doit proposer beaucoup plus. Il est vrai que l'assistante médicale effectue une grande partie de son travail, en particulier en ce qui concerne le patient, selon la philosophie et les directives du cabinet où elle travaille. Il est tout aussi vrai que le travail d'équipe inhérent à un cabinet ne peut être enseigné et compris qu'au cabinet. Ce point a influé sur la conception didactique de l'aide-mémoire.
Quels sont les points forts de l'aide-mémoire? Ses points forts concernent principalement le travail d'équipe et les interfaces entre le travail du médecin et celui des assistantes médicales ainsi que leurs compétences en matière de conseil. Cette approche s'est imposée pour deux raisons: premièrement, l'importante réglementation législative pousse à réfléchir en équipe et à assumer une responsabilité commune dans les tâches quotidiennes au cabinet et deuxièmement la prise en charge des malades chroniques dans les cabinets de médecine de premier recours gagnera en importance ces prochaines années. Dans ce domaine, les assistantes médicales sont appelées à jouer un rôle déterminant pour épauler le médecin. 
Le cabinet médical est au centre du nouvel aide-mémoire en qualité de lieu de formation.

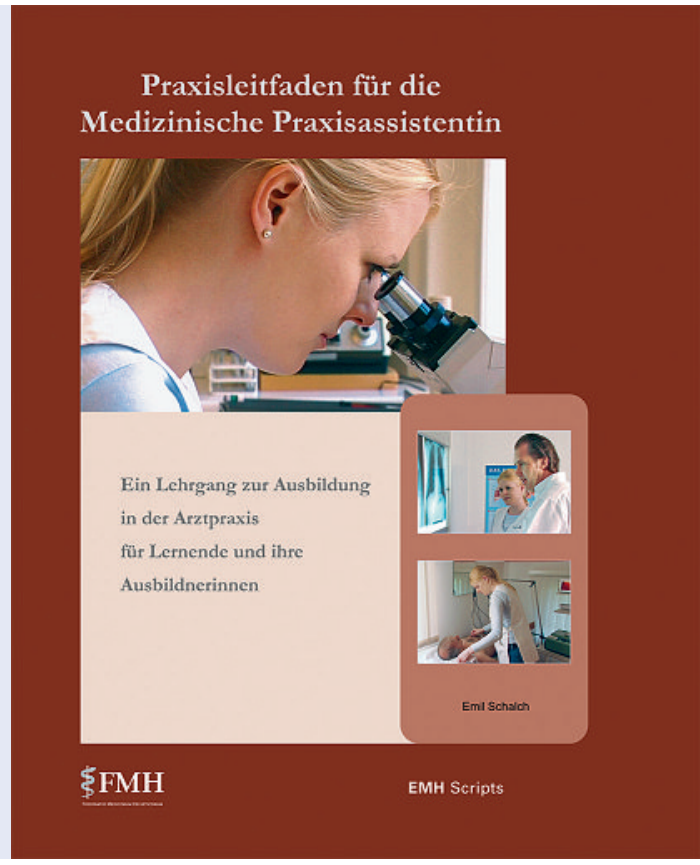

L'aide-mémoire s'adresse tant aux assistantes qu'aux formateurs. Quels sont les avantages pour l'un et l'autre? Le formateur y trouve des directives lui indiquant quels sujets importants pour le cabinet médical il doit transmettre à l'apprenante et de quelle manière. Il peut choisir un chapitre comme «thème hebdomadaire» et l'étudier pendant une semaine avec l'apprenante pour acquérir les connaissances et/ou la manière d'agir qui s'y trouvent décrites. Il trouvera également en annexe des outils pédagogiques visant à documenter les connaissances acquises.

L'apprenant peut et doit utiliser l'aide-mémoire comme un manuel et comme un outil pour répéter ses cours. Dans la partie générale, il y trouvera un guide pour les «3 lieux d'apprentissage» que sont le cabinet médical, l'école professionnelle et les cours interentreprises ainsi que les tâches spécifiques qui en découlent. L'aide-mémoire fournit en outre des conseils pour apprendre et travailler avec le patient dans un cabinet de praticien. Après quelques indications de la part du formateur, l'apprenant devrait être en mesure d'aborder seul les différents chapitres.

\section{Qu'est-ce qui vous a incité à créer cet aide-mémoire?}

Les offices de formation professionnelle et les écoles professionnelles exigent depuis longtemps déjà la création d'un guide de formation pour les cabinets médicaux, car les différents contenus de formation et les procédures propres au cabinet et à la prise en charge des patients ne peuvent être enseignés qu'au cabinet.
Lorsqu'on dirige un grand cabinet médical à la campagne, on ne pense pas nécessairement à écrire un livre. Mais il s'est avéré que dans le cadre de la répartition des tâches au sein de la commission de réforme, j'ai été mandaté pour rédiger cet aide-mémoire. J'ai aussi eu la chance de pouvoir choisir comment je souhaitais répartir mon temps de travail. Le fait que je m'étais déjà familiarisé avec le déroulement du travail et les tâches des assistantes médicales lors de mes activités en faveur de la qualité dans mon cabinet a aussi joué un rôle dans cette décision.

Dans quelle mesure avez-vous personnellement pu tirer profit de ce travail? Qu'est-ce qui vous a particulièrement inspiré?

J'ai été inspiré par le travail de mon équipe et par la constatation que seul un plus grand travail en commun et la délégation de certaines tâches à mes assistantes m'ont permis de dégager le temps nécessaire pour la rédaction. L'importance du travail d'équipe s'est ensuite très clairement reflétée dans l'aide-mémoire. Etant donné que la délégation des tâches médicales aux assistantes n'est pas prévue par le TARMED, l'avantage que je retire de ce travail de rédaction est essentiellement humain. J'ai apprécié, en cette période de surcharge chronique, la sollicitude de mes assistantes, la sympathie de mes patients et le soutien des membres de la commission de réforme et du Comité central de la FMH. Je suis très content de voir que j'ai pu réaliser beaucoup plus de choses que je ne l'aurais cru.

\section{Aide-mémoire et documentation pédagogique à commander}

Le nouvel Aide-mémoire pour les assistantes médicales comporte environ 400 pages regroupées dans un classeur que vous pouvez commander auprès des Editions médicales suisses pour la somme de 98 CHF (sans frais de port ni TVA) par téléphone au 06146785 75, par fax au 061 4678576 ou par courriel à auslieferung@emh.ch. Ce classeur sera disponible dès la mi-août 2010 en allemand. Une traduction française est prévue.

La version allemande de la nouvelle documentation pédagogique pour les assistantes médicales (anciennement Manuel de travail) peut être commandée auprès du secrétariat de l'Association suisse des assistantes médicales (SVA), ainsi qu'auprès du Secrétariat général de la FMH: info@sva.ch, www.sva.ch et mpa@fmh.ch, www.fmh.ch. Le classeur coûte 20 CHF (sans frais de port ni TVA); les versions française et italienne seront disponibles dès la mi-août 2010. 